Journal of Mathematics and Informatics

Vol. 17, 2019, 1-8

ISSN: 2349-0632 (P), 2349-0640 (online)

Published 20 May 2019

www. researchmathsci.org

DOI: http://dx.doi.org/10.22457/jmi.141av17al

Journal of

Mathematics and

Informatics

\title{
A Generalization of Hyperbolic Special Mean and its Schur Power Convexity
}

\author{
Chun-yong Wang ${ }^{1}$, Xiaoqiang Zhou ${ }^{2}$ and Yameng Deng ${ }^{2}$ \\ ${ }^{1}$ School of Mathematics and Computer Science, \\ Hezhou University Hezhou, Guangxi, 542800, P.R.China \\ ${ }^{2}$ School of Information Science and Engineering \\ Hunan Institute of Science and Technology \\ Yueyang, Hunan, 414006, P.R. China \\ ${ }^{2}$ Corresponding author. Email: zxq0923@163.com
}

Received 22 April 2019; accepted 17 May 2019

Abstract. By combining hyperbolic special mean with power average, a more general mean is studied. The necessary and sufficient conditions for the determination of Schur are also given.

Keywords: Hyperbolic function; Schur convexity; Schur power convexity

AMS Mathematics Subject Classification (2010): 52A55

\section{Introduction}

Let $x, y$ be two positive numbers, then

$$
\begin{gathered}
M_{p}(x, y)=\left\{\begin{array}{cc}
\left(\frac{x^{p}}{2}+\frac{y^{p}}{2}\right)^{1 / p}, p \neq 0 \\
\sqrt{x y}, & p=0
\end{array}\right. \\
G(x, y)=M_{0}(x, y)=\sqrt{x y}, \\
A(x, y)=M_{1}(x, y)=\frac{x+y}{2}
\end{gathered}
$$

are called Power mean, geometric mean and arithmetic mean of $x, y$, respectively.

In 2003, American mathematical monthly, problem 11031, proposed a strong mean and Inequality conjecture as below.

Problem 11031: Let $x, y>0$, define $M(x, y)=\ln N(x, y)$, where

$$
N=N(x, y)=\frac{1+\ln (\sqrt{1+f}+\sqrt{f})}{1-\ln (\sqrt{1+f}-\sqrt{f})}, f=f(x, y)=\frac{1}{4}\left(e^{\frac{2\left(e^{x}-1\right)}{e^{x}+1}}-1\right)\left(e^{\frac{2\left(e^{y}-1\right)}{e^{y}+1}}-1\right) e^{-\left(\frac{e^{x}-1}{e^{x}+1}+\frac{e^{y}-1}{e^{y}+1}\right)},
$$

to prove or disprove $M(x, y) \leq G(x, y)$. 
Chunyong Wang, Xiaoqiang Zhou and Yameng Deng

Zhang [14] studied the Schur- geometric convexity of $m(x, y)=\sqrt{1+f(x, y)}+\sqrt{f(x, y)}$, and give the positive proof of the above problem.

Li and Shi [6] adapted $M(x, y)$ as $M(x, y)=2 \operatorname{th}^{-1} \operatorname{sh}^{-1} \sqrt{\operatorname{sh}\left(\operatorname{th} \frac{x}{2}\right) \operatorname{sh}\left(\text { th } \frac{y}{2}\right)}$, then by geometric convexity of $\operatorname{sh}(\operatorname{th} x)$, they also solved the above Inequality conjecture.

Shi [7] discussed the Schur- convexity and Schur- geometric convexity of $M(x, y)$ He [5] further defined $H(x, y)=2 \tan ^{-1} \sin ^{-1} \sqrt{\sin \left(\tan \frac{x}{2}\right) \sin \left(\tan \frac{y}{2}\right)}\left(x, y \in\left(0,2 \tan ^{-1} \pi / 2\right)\right)$, by polynomial discriminant system [9-10], discussed the Schur power convexity of $M(x, y)$ and $H(x, y)[2,11-13,17]$.

Similarly, Chen et. al. [1] defined

$$
\begin{gathered}
M^{*}(x, y)=2 \operatorname{sh}^{-1} \operatorname{th}^{-1} \sqrt{\operatorname{th}\left(\operatorname{sh} \frac{x}{2}\right) \operatorname{th}\left(\operatorname{sh} \frac{y}{2}\right)}(x, y \in(0,+\infty)), \\
H^{*}(x, y)=2 \sin ^{-1} \tan ^{-1} \sqrt{\tan \left(\sin \frac{x}{2}\right) \tan \left(\sin \frac{y}{2}\right)}(x, y \in(0, \pi)),
\end{gathered}
$$

and then discussed their Schur power convexity.

In this paper, we generalize the above means and define

$$
\begin{aligned}
M_{p}^{*}(x, y) & =2 \operatorname{sh}^{-1} \operatorname{th}^{-1}\left[M_{p}\left(\operatorname{th}\left(\operatorname{sh} \frac{x}{2}\right), \operatorname{th}\left(\operatorname{sh} \frac{y}{2}\right)\right)\right] \\
& =\left\{\begin{array}{ll}
2 \operatorname{sh}^{-1} \operatorname{th}^{-1}\left\{\left[\frac{1}{2} \operatorname{th}^{p}\left(\operatorname{sh} \frac{x}{2}\right)+\frac{1}{2} \operatorname{th}^{p}\left(\operatorname{sh} \frac{y}{2}\right)\right]^{1 / p}\right\}, & p>0 \\
2 \operatorname{sh}^{-1} \operatorname{th}^{-1} \sqrt{\operatorname{th}\left(\operatorname{sh} \frac{x}{2}\right) \operatorname{th}\left(\operatorname{sh} \frac{y}{2}\right),} & p=0
\end{array}(x, y \in(0,+\infty)),\right.
\end{aligned}
$$

then discussed its Schur power convexity.

\section{Definition and lemma}

For $x=\left(x_{1}, x_{2}, \cdots, x_{n}\right) \in R^{n}$, We rearrange its components in descending order, and denote $x_{[1]} \geq x_{[2]} \geq \cdots \geq x_{[n]}$. When $x_{i} \leq y_{i}(i=1, \cdots, n)$, we write $x \leq y$ for short.

Definition 1. [8] Suppose $x, y \in R^{n}$ satisfy:

(i) $\sum_{i=1}^{k} x_{[i]} \leq \sum_{i=1}^{k} y_{[i]}(k=1,2, \cdots, n-1)$,

(ㅁ) $\sum_{i=1}^{n} x_{i}=\sum_{i=1}^{n} y_{i}$,

then we say $x$ is controlled by $y$, denoted by $x \prec y$.

Definition 2. [8] Suppose $\Omega \subset R^{n}, \varphi: \Omega \rightarrow R$, 
A Generalization of Hyperbolic Special Mean and its Schur Power Convexity

(i) If for any $x, y \in \Omega, x \leq y \Rightarrow \varphi(x) \leq \varphi(y)$, then $\varphi$ is called increasing function on $\Omega$; if $-\varphi$ is a increasing function on $\Omega$, then $\varphi$ is called reduction function on $\Omega . \varphi$ is called Schur convex function on $\Omega$

(ii) If for any $x, y \in \Omega, x \prec y \Rightarrow \varphi(x) \leq \varphi(y)$, then $\varphi$ is called Schur-convex function on $\Omega$; if $-\varphi$ is called Schur-convex function on $\Omega$, then $\varphi$ is called Schur-concave function on $\Omega$.

Lemma 1. [8] Let $E\left(\subseteq R^{n}\right)$ be a symmetric convex set with certain interior points, $f: E \rightarrow R$ is continuous and differentiable in int $E$, then $f$ is called a Schur- convex (concave) function on $E$ if and only iff $f$ is symmetrical on $E$ and for all $x \in \operatorname{int} E$,

$$
\left(x_{1}-x_{2}\right)\left(\frac{\partial f}{\partial x_{1}}-\frac{\partial f}{\partial x_{2}}\right) \geq 0(\leq 0) \text {. }
$$

Definition 3. [14] Let $E \subseteq R_{++}^{n}$,For any two-vector $x, y \in E$, when $\left(\ln x_{1}, \ln x_{2}, \cdots, \ln x_{n}\right) \prec\left(\ln y_{1}, \ln y_{2}, \cdots, \ln y_{n}\right)$, there are $f(x) \leq f(y)$. Then $\mathrm{f}$ are the Schurgeometric convex function on $\mathrm{E}$; $\mathrm{f}$ is the Schur- geometry concave function on $\mathrm{E}$, if and only if-f is schur-geometric convex function.

Lemma 2. [16] Let $E\left(\subseteq R^{n}\right)$ is a symmetric set with interior points,

$\left\{\left(\ln x_{1}, \ln x_{2}, \cdots, \ln x_{n}\right) \mid x \in E\right\}$ is convex set, $f: E \rightarrow R$ continuation, and differentiable in the int $\mathrm{E}$. Then the necessary and sufficient condition for $\mathrm{f}$ to be a convex (concave) function of Schur- geometry is $f$ is symmetric on E, and for all $x \in \operatorname{int} E$, both of

$$
\left(x_{1}-x_{2}\right)\left(x_{1} \frac{\partial f}{\partial x_{1}}-x_{2} \frac{\partial f}{\partial x_{2}}\right) \geq(\leq) 0
$$

Definition 4. [3,4] Let $E \subset R_{++}^{n}, f: E \rightarrow R$,If you take it at will $x, y \in E$, when

$$
\left(\frac{1}{x_{1}}, \frac{1}{x_{2}}, \cdots, \frac{1}{x_{n}}\right) \prec\left(\frac{1}{y_{1}}, \frac{1}{y_{2}}, \cdots, \frac{1}{y_{n}}\right),
$$

there are $f(x) \leq f(y)$, Then $\mathrm{f}$ are the Schur- harmonic convex function on E; If $-\mathrm{f}$ is harmonic convex function on $\mathrm{E}$,then $\mathrm{f}$ are the Schur- harmonic concave function on $\mathrm{E}$.

Lemma 3. [4] Let $E\left(\subset R_{++}^{n}\right)$ is a symmetric set with interior points, $\left\{\left(1 / x_{1}, 1 / x_{2}, \cdots, 1 / x_{n}\right) \mid x \in E\right\}$ is convex set, $f: E \rightarrow R$ continuation, and differentiable in the int $\mathrm{E}$, Then the necessary and sufficient condition for $\mathrm{f}$ to be a convex (concave) function of Schur- harmonic is $f$ is symmetric on $\mathrm{E}$, and for all $x \in \operatorname{int} E$, both of

$$
\left(x_{1}-x_{2}\right)\left(x_{1}^{2} \frac{\partial \varphi}{\partial x_{1}}-x_{2}^{2} \frac{\partial \varphi}{\partial x_{2}}\right) \geq 0(\leq 0) .
$$


Chunyong Wang, Xiaoqiang Zhou and Yameng Deng

Definition 5. [11-13] (i) Let $f: R_{++} \rightarrow R$ is strictly monotone function, $\Omega \subset R^{n}$. If for any $x, y \in \Omega$,always $f^{-1}(\alpha f(x)+\beta f(y)) \in \Omega$, call $\Omega$ is $f$-convex set, among $\alpha, \beta \in[0,1]$ and $\alpha+\beta=1$.

(ii) Let $\Omega \subset R^{n}, \Omega$ internal is not empty. $\varphi: \Omega \rightarrow R$, for any $x, y \in \Omega$, when $f(x) \prec f(y)$ there are $\varphi(x) \leq \varphi(y)$, Then $-\varphi$ are $\Omega$ the Schur- $\mathbf{f}$ convex function on E. If $-\varphi$ is Schur- $f$ convex function on $\Omega$, then $\varphi$ are the Schur- $f$ concave function on $\Omega$.

According to the definition of Schur- $f$ convex function. If $\mathrm{g}$ is monotonously increasing (decrease), $g(\varphi(x))$ make sense, then $\varphi$ is Schur- $f$ convex function, if and only if $g \circ \varphi$ is Schur- $f$ convex (concave) function.

Definition 6. [11-13] In definition 5, we take

$$
f: x \in(0,+\infty) \rightarrow \begin{cases}\frac{x^{m}-1}{m}, & m \neq 0 ; \\ \ln x, & m=0 .\end{cases}
$$

then $\varphi$ are the Schur- $m$ order power convex function on $\Omega$; If $-\varphi$ is Schur- $m$ order power convex function on $\Omega$, then $\varphi$ are the Schur- $m$ order power concave function on $\Omega$.

Lemma 4. [11-13] Let $f: R \rightarrow R$ is strictly monotone differentiable functions, $\Omega\left(\subset R_{++}^{n}\right)$ is symmetry with interior points $f$-convex set, $\varphi: \Omega \rightarrow R$ on $\Omega$ is continuation, Inside of $\Omega, \Omega^{0}$ is differentiable, then the necessary and sufficient condition for $\varphi$ to be a Schur$f$ bulge (Schur- $f$ concave) is $\varphi$ is symmetric on $\Omega$, and for $\forall x \in \Omega^{0}$, we have

$$
\Delta:=\left(f\left(x_{1}\right)-f\left(x_{2}\right)\right)\left(\frac{1}{f^{\prime}\left(x_{1}\right)} \frac{\partial \varphi}{\partial x_{1}}-\frac{1}{f^{\prime}\left(x_{2}\right)} \frac{\partial \varphi}{\partial x_{2}}\right) \geq 0(\leq 0) .
$$

For Schur- $m$ order power convex function, if $m \neq 0$, the corresponding Schur condition is

$$
\frac{x_{1}^{m}-x_{2}^{m}}{m}\left(x_{1}^{1-m} \frac{\partial \varphi}{\partial x_{1}}-x_{2}^{1-m} \frac{\partial \varphi}{\partial x_{2}}\right) \geq 0(\leq 0) .
$$

It's not hard to find, formula (4) Synthetic formulae (1-5).

Remarks : Owing to $\operatorname{sgn}\left(\frac{x_{1}^{m}-x_{2}^{m}}{m}\right)=\operatorname{sgn}\left(x_{1}-x_{2}\right)$, so the above Schur condition is equivalent to

$$
\left(x_{1}-x_{2}\right)\left(x_{1}^{1-m} \frac{\partial \varphi}{\partial x_{1}}-x_{2}^{1-m} \frac{\partial \varphi}{\partial x_{2}}\right) \geq 0(\leq 0)(m \in R) .
$$

Lemma 5. $g_{1}(x)=x^{-1} \operatorname{th}\left(\operatorname{sh} \frac{x}{2}\right)$ on $(0,+\infty)$ is monotone decreasing, and $0<g_{1}(x)<\frac{1}{2}$. 
A Generalization of Hyperbolic Special Mean and its Schur Power Convexity

Proof: $g_{1}^{\prime}(x)=-\frac{1}{x^{2}}\left\{\operatorname{th}\left(\operatorname{sh} \frac{x}{2}\right)-\frac{1}{2} x\left[1-\operatorname{th}^{2}\left(\operatorname{sh} \frac{x}{2}\right)\right] \operatorname{ch} \frac{x}{2}\right\}=-\frac{2 \operatorname{sh}\left(\operatorname{sh} \frac{x}{2}\right) \operatorname{ch}\left(\operatorname{sh} \frac{x}{2}\right)-x \operatorname{ch} \frac{x}{2}}{2 x^{2} \operatorname{ch}^{2}\left(\operatorname{sh} \frac{x}{2}\right)}$, owing to $p_{1}(t)=\operatorname{sh} t, p_{2}(t)=\operatorname{ch} t$ all about $t$ on $(0,+\infty)$ is monotone increment, and $\operatorname{sh} t>t(t>0) t$, then

$$
2 \operatorname{sh}\left(\operatorname{sh} \frac{x}{2}\right) \operatorname{ch}\left(\operatorname{sh} \frac{x}{2}\right)-x \operatorname{ch} \frac{x}{2}>2 \operatorname{sh} \frac{x}{2} \operatorname{ch} \frac{x}{2}-x \operatorname{ch} \frac{x}{2}>2 \times \frac{x}{2} \operatorname{ch} \frac{x}{2}-x \operatorname{ch} \frac{x}{2}=0 .
$$

Thus $g_{1}^{\prime}(x)<0, g_{1}(x)$ about $x$ on $(0,+\infty)$ is monotone decrease. Obvious $g_{1}(x)>0$, again

$$
\lim _{x \rightarrow 0^{+}} g_{1}(x)=\lim _{x \rightarrow 0^{+}} \frac{\operatorname{th}\left(\operatorname{sh} \frac{x}{2}\right)}{x}=\lim _{x \rightarrow 0^{+}} \frac{\operatorname{sh} \frac{x}{2}}{x}=\lim _{x \rightarrow 0^{+}} \frac{\frac{x}{2}}{x}=\frac{1}{2} .
$$

Then for any $x \in(0,+\infty)$, we have $g_{1}(x)<g_{1}(0)=\frac{1}{2}$.

Lemma 6. [1] $g_{2}(x)=2 x \operatorname{ch} \frac{x}{2}\left[\operatorname{sh}\left(2 \operatorname{sh} \frac{x}{2}\right)\right]^{-1}$ on $(0,+\infty)$ is monotone decrease, and $0<g_{2}(x)<2$.

\section{Main results and proof}

Theorem 1. $M_{p}^{*}(x, y)$ about $(x, y)$ on $(0,+\infty)^{2}$ Schur-m order concave, if and only if $m \geq p$.

Proof: When $p=0, M_{p}^{*}(x, y)=M^{*}(x, y)$, on document[12] certified $M^{*}(x, y)$ about $(x, y)$ on $(0,+\infty)^{2}$ Schur-m order concave, if and only if $m \geq p$.

When $p>0$,calculated

Among

$$
\begin{aligned}
& \frac{\partial M_{p}^{*}(x, y)}{\partial x}=\frac{f_{1}(x, y)}{f_{2}(x, y)} \operatorname{th}^{p-1}\left(\operatorname{sh} \frac{x}{2}\right)\left[1-\operatorname{th}^{2}\left(\operatorname{sh} \frac{x}{2}\right)\right] \operatorname{ch} \frac{x}{2}, \\
& \frac{\partial M_{p}^{*}(x, y)}{\partial y}=\frac{f_{1}(x, y)}{f_{2}(x, y)} \operatorname{th}^{p-1}\left(\operatorname{sh} \frac{y}{2}\right)\left[1-\operatorname{th}^{2}\left(\operatorname{sh} \frac{y}{2}\right)\right] \operatorname{ch} \frac{y}{2} .
\end{aligned}
$$

$$
\begin{gathered}
f_{1}(x, y)=\frac{\left[\frac{1}{2} \operatorname{th}^{p}\left(\operatorname{sh} \frac{x}{2}\right)+\frac{1}{2} \operatorname{th}^{p}\left(\operatorname{sh} \frac{y}{2}\right)\right]^{1 / p-1}}{2 \sqrt{1+\left\{\operatorname{th}^{-1}\left[\left(\frac{1}{2} \operatorname{th}^{p}\left(\operatorname{sh} \frac{x}{2}\right)+\frac{1}{2} \operatorname{th}^{p}\left(\operatorname{sh} \frac{y}{2}\right)\right)^{1 / p}\right]\right\}^{2}}}, \\
f_{2}(x, y)=1-\left[\left(\frac{1}{2} \operatorname{th}^{p}\left(\operatorname{sh} \frac{x}{2}\right)+\frac{1}{2} \operatorname{th}^{p}\left(\operatorname{sh} \frac{y}{2}\right)\right)^{1 / p}\right]^{2} .
\end{gathered}
$$

Obvious $f_{1}(x, y)>0$, owing to $\operatorname{th}\left(\operatorname{sh} \frac{x}{2}\right), \operatorname{th}\left(\operatorname{sh} \frac{y}{2}\right) \in(0,1)$, then 
Chunyong Wang, Xiaoqiang Zhou and Yameng Deng

$$
\operatorname{th}^{p}\left(\operatorname{sh} \frac{x}{2}\right), \operatorname{th}^{p}\left(\operatorname{sh} \frac{y}{2}\right) \in(0,1),\left(\frac{1}{2} \operatorname{th}^{p}\left(\operatorname{sh} \frac{x}{2}\right)+\frac{1}{2} \operatorname{th}^{p}\left(\operatorname{sh} \frac{y}{2}\right)\right)^{1 / p} \in(0,1),
$$

Thus $f_{2}(x, y)>0$.

$$
\begin{aligned}
& \Delta_{M_{p}^{*}}(x, y)=(x-y)\left(x^{1-m} \frac{\partial M_{p}^{*}(x, y)}{\partial x}-y^{1-m} \frac{\partial M_{p}^{*}(x, y)}{\partial y}\right) \text {, then } \\
& \Delta_{M_{p}^{*}}(x, y) \\
= & \frac{(x-y) f_{1}(x, y)}{f_{2}(x, y)}\left\{x^{1-m} \operatorname{th}^{p-1}\left(\operatorname{sh} \frac{x}{2}\right)\left[1-\operatorname{th}^{2}\left(\operatorname{sh} \frac{x}{2}\right)\right] \operatorname{ch} \frac{x}{2}-y^{1-m} \operatorname{th}^{p-1}\left(\operatorname{sh} \frac{y}{2}\right)\left[1-\operatorname{th}^{2}\left(\operatorname{sh} \frac{y}{2}\right)\right] \operatorname{ch} \frac{y}{2}\right\} \\
= & \frac{(x-y)^{2} f_{1}(x, y)}{f_{2}(x, y)} \cdot \frac{x^{1-m} \operatorname{th}^{p-1}\left(\operatorname{sh} \frac{x}{2}\right)\left[1-\operatorname{th}^{2}\left(\operatorname{sh} \frac{x}{2}\right)\right] \operatorname{ch} \frac{x}{2}-y^{1-m} \operatorname{th}^{p-1}\left(\operatorname{sh} \frac{y}{2}\right)\left[1-\operatorname{th}^{2}\left(\operatorname{sh} \frac{y}{2}\right)\right] \operatorname{ch} \frac{y}{2}}{x-y}
\end{aligned}
$$

owing to

$$
\begin{aligned}
x^{1-m} \operatorname{th}^{p-1}\left(\operatorname{sh} \frac{x}{2}\right)\left[1-\operatorname{th}^{2}\left(\operatorname{sh} \frac{x}{2}\right)\right] \operatorname{ch} \frac{x}{2} & =x^{p-m}\left[x^{-1} \operatorname{th}\left(\operatorname{sh} \frac{x}{2}\right)\right]^{p} \frac{x\left[1-\operatorname{th}^{2}\left(\operatorname{sh} \frac{x}{2}\right)\right] \operatorname{ch} \frac{x}{2}}{\operatorname{th}\left(\operatorname{sh} \frac{x}{2}\right)} \\
& =x^{p-m}\left[x^{-1} \operatorname{th}\left(\operatorname{sh} \frac{x}{2}\right)\right]^{p} \frac{2 x \operatorname{ch} \frac{x}{2}}{\operatorname{sh}\left(2 \operatorname{sh} \frac{x}{2}\right)} \\
& =x^{p-m}\left[g_{1}(x)\right]^{p} g_{2}(x) .
\end{aligned}
$$

When $m \geq p$, apparently $x^{p-m}>0$, and $y_{1}=x^{p-m}$ about $x$ on $(0,+\infty)$ is monotone decrease. By Lemma 5 and $p>0$, we have $\left[g_{1}(x)\right]^{p}>0$ and $y_{2}=\left[g_{1}(x)\right]^{p}$ about $x$ on $(0,+\infty)$ is monotone decrease. Then according to lemma 6 , obvious $g_{2}(x)>0$, and $g_{2}(x)$ about $x$ on $(0,+\infty)$ is monotone decrease. Comprehensive, function $h(x)=x^{p-m}\left[g_{1}(x)\right]^{p} g_{2}(x)$ about $x$ on $(0,+\infty)$ is monotone decrease, then

$$
\Delta_{M_{p}^{*}}(x, y)=\frac{(x-y)^{2} f_{1}(x, y)}{f_{2}(x, y)} \cdot \frac{h(x)-h(y)}{x-y} \leq 0 .
$$

According to lemma 6 , we can get $M_{p}^{*}(x, y)$ about $(x, y)$ on $(0,+\infty)^{2}$ is Schur-m order concave.

When $p>0$ and $m<p$,owing to

$$
\begin{gathered}
\lim _{x \rightarrow+\infty}\left[g_{1}(x)\right]^{p}=\left[\lim _{x \rightarrow+\infty} g_{1}(x)\right]^{p}=\left(\lim _{x \rightarrow+\infty} \frac{1}{x}\right)^{p}=0, \\
0<\frac{2 x^{1+p-m} \operatorname{ch} \frac{x}{2}}{\operatorname{sh}\left(2 \operatorname{sh} \frac{x}{2}\right)}<\frac{2 x^{1+p-m} \operatorname{ch} \frac{x}{2}}{\operatorname{sh} x}=\frac{x^{1+p-m}}{\operatorname{sh} \frac{x}{2}}<\frac{2 x^{1+p-m}}{e^{\frac{x}{2}}-1} \rightarrow 0(x \rightarrow+\infty) .
\end{gathered}
$$


A Generalization of Hyperbolic Special Mean and its Schur Power Convexity

Then $\lim _{x \rightarrow+\infty} h(x)=\lim _{x \rightarrow+\infty}\left[g_{1}(x)\right]^{p} \cdot \lim _{x \rightarrow+\infty} \frac{2 x^{1+p-m} \operatorname{ch} \frac{x}{2}}{\operatorname{sh}\left(2 \operatorname{sh} \frac{x}{2}\right)}=0 \times 0=0$.

So $\quad \lim _{x \rightarrow+\infty}[h(x)-h(1)]=\lim _{x \rightarrow+\infty}\left[h(x)-\operatorname{th}^{p}\left(\operatorname{sh} \frac{1}{2}\right) \frac{2 \operatorname{ch} \frac{1}{2}}{\operatorname{sh}\left(2 \operatorname{sh} \frac{1}{2}\right)}\right]=-\operatorname{th}^{p}\left(\operatorname{sh} \frac{1}{2}\right) \frac{2 \operatorname{ch} \frac{1}{2}}{\operatorname{sh}\left(2 \sin \frac{1}{2}\right)}<0$.

Then $\exists x_{0} \in(1,+\infty)$ make $\frac{h\left(x_{0}\right)-h(1)}{x_{0}-1}<0, \Delta_{M_{p}^{*}}\left(x_{0}, 1\right)<0$.

Associative Lemma 5 and Lemma 6, we can get

$$
0<h(y)=y^{p-m}\left[g_{1}(y)\right]^{p} g_{2}(y)<y^{p-m}\left(\frac{1}{2}\right)^{p} \times 2 \rightarrow 0\left(y \rightarrow 0^{+}\right) .
$$

Then $\lim _{y \rightarrow 0^{+}} h(y)=0$, so

$$
\lim _{y \rightarrow 0^{+}}[h(1)-h(y)]=\lim _{y \rightarrow 0^{+}}\left[\operatorname{th}^{p}\left(\operatorname{sh} \frac{1}{2}\right) \frac{2 \operatorname{ch} \frac{1}{2}}{\operatorname{sh}\left(2 \operatorname{sh} \frac{1}{2}\right)}-h(y)\right]=\operatorname{th}^{p}\left(\operatorname{sh} \frac{1}{2}\right) \frac{2 \operatorname{ch} \frac{1}{2}}{\operatorname{sh}\left(2 \operatorname{sh} \frac{1}{2}\right)}>0
$$

Then $\exists y_{0} \in(0,1)$ make $\frac{h(1)-h\left(y_{0}\right)}{1-y_{0}}>0, \Delta_{M_{p}^{*}}\left(1, y_{0}\right)>0$.

Because at this point, $\Delta_{M_{p}^{*}}(x, y)$ on $(0,+\infty)^{2}$ is symbol uncertainty, thus $M_{p}^{*}(x, y)$ is not $(0,+\infty)^{2}$ Schur-m power concave (convex) function.

In summary, the theorem can be proved.

\section{Two unresolved issues}

Question 1. To average $M_{p}^{*}(x, y)$, Where the range of values of $p$ can be extended to $R$ try to give $M_{p}^{*}(x, y)(p \in R)$ about $(x, y)$ on $(0,+\infty)^{2}$ necessary and sufficient conditions for power convexity of Schur-m order.

Question 2. Similarly the form of $H^{*}(x, y)$. More general averages involving trigonometric functions can be defined as follows

$$
\begin{aligned}
H_{p}^{*}(x, y) & =2 \sin ^{-1} \tan ^{-1}\left[M_{p}\left(\tan \left(\sin \frac{x}{2}\right), \tan \left(\sin \frac{y}{2}\right)\right)\right] \\
& =\left\{\begin{array}{ll}
2 \sin ^{-1} \tan ^{-1}\left\{\left[\frac{1}{2} \tan ^{p}\left(\sin \frac{x}{2}\right)+\frac{1}{2} \tan ^{p}\left(\sin \frac{y}{2}\right)\right]^{1 / p}\right\}, & p \neq 0 \\
2 \sin ^{-1} \tan ^{-1} \sqrt{\tan \left(\sin \frac{x}{2}\right) \tan \left(\sin \frac{y}{2}\right)}, & p=0
\end{array}(x, y \in(0, \pi)),\right.
\end{aligned}
$$

try to give $H_{p}^{*}(x, y)$ about $(x, y)$ on $(0, \pi)^{2}$ necessary and sufficient conditions for power convexity of Schur-m order. 
Chunyong Wang, Xiaoqiang Zhou and Yameng Deng

Acknowledgments. This work was supported by the Doctor's Scientific Research Foundation of Hezhou University (No.HZUBS201505) and Natural Science Foundation of Hunan Province (No: 2019JJ40100). Also, we are grateful to the reviewers for their valuable comments.

\section{REFERENCES}

1. D.S.Chen, C.Y.Wang and S.D.Tao, Two new "strange" averages and their Schur power convexity, Mathematical Practice and Cognition (In press).

2. Y.P.Deng, S.H.Wu and D.He, On the Schur power convexity of generalized muirhead mean, Practice and Cognition of Mathematics (Chinese), 44(5) (2014) 255-268.

3. K.Z.Guan, Schur-convexity of the complete symmetric function, Math. Inequal. Appl., 9 (2006) 567-576.

4. K.Z.Guan, Some properties of a class of symmetric functions, J. Math. Anal. Appl., 336 (2007) 70-80.

5. D.He, On the Schur power convexity of two "peculiar" averages, Journal of Guangdong second normal University (Chinese), 36(3) (2016) 30-38.

6. D.M.Li and H.N.Shi. A New proof of the conjecture of a binary mean value inequality, Mathematical Practice and Cognition (Chinese), 36(4) (2006) 278-283.

7. H.N.Shi, Controlled theory and analytic inequalities, Harbin: Harbin University of Technology Press (Chinese) (2012) 283-287.

8. B.Y.Wang, Basis of control inequality, Beijing: Beijing normal University Press (Chinese), 1990.

9. L.Yang, J.Z.Zhang and X.R.Hou, Nonlinear algebraic equations and machine proof of theorems, Shanghai: Shanghai Science and Technology Education Press (Chinese), (1996) 137-166.

10. L.Yang and B.C.Xia, Inequality machine proof and automatic discovery, Beijing: Science Publishing House (Chinese), (2008) 33-46.

11. Z.H.Yang, Schur power convexity of Stolarsky means, Publ. Math. Debrecen, 80 (2012) 43-66.

12. Z.H.Yang, Schur power convexity of Gini means, Bull. Korean Math. Soc., 50(2) (2013) 485-498.

13. Z.H.Yang, Schur power convexity of Daróczy means, Math. Inequal. Appl., 16(3) (2013) 751-762.

14. X.M.Zhang, Geometric convex function, Hefei: Anhui University Press (Chinese), (2004) 107-121.

15. X.M.Zhang and T.Q.Xu, Definition and Application of Generalized SCHURgeometric Convex function, Journal of Qingdao Vocational and Technical College (Chinese), 18(4) (2005) 60-63.

16. X.M.Zhang and Y.M Chu, New theory of analytic inequalities, Harbin: Harbin Gonglai Publishing House (Chinese), (2009) 93-94.

17. X.M.Zhang. The Schur-product of several $\mathrm{n}-\mathrm{p}$ element averages order power convexity, Journal of Hunan Institute of Technology (Chinese), 24 (2011) 1-7. 